\title{
Schulung für Frauen mit Mamma-Ca
}

Frauen mit Diagnose Brustkrebs erleben eine weite Spanne körperlicher, psychologischer, Informationsbezogener und Unterstützungs-Bedürfnisse, die häufig weit über die ersten Wochen nach der Operation hinaus bestehen bleiben. Das Akzeptieren der Diagnose, Behandlungsentscheidungen, emotionaler Stress, der mit der körperlichen Veränderung und dem Verlust assoziiert ist, Änderungen im Lebensstil, Unsicherheit und existentielle Sorgen erzeugen einen Bedarf an Information und Unterstützung, der während allen Phasen der Brustkrebserkrankung anhält.

In der Literatur wurden Schlüsselphasen der Brustkrebserfahrung identifiziert: Diagnose, post-OP-Phase, adjuvante Therapie und fortschreitende Genesung. Wenn die Bedürfnisse einer Phase nicht ausreichend erfüllt werden, besteht die Gefahr, dass sie die Bedürfnisse der nächsten Phase überlagern. Daher ist es wichtig phasengerecht $\mathrm{zu}$ intervenieren, um die physischen, emotionalen und sozialen Bedürfnisse zu erfüllen und den Anpassungsprozess zu optimieren. Budin et al. untersuchten den Beitrag der phasenspezifischen Psychoedukation und der Telephonberatung (Telephone Counseling = TC) im fortlaufenden Prozess der Anpassung bei Patientinnen mit Brustkrebs.

Brustkrebs betrifft nicht nur den $\mathrm{Pa}$ tienten, sondern auch die Familie, speziell den Partner mit allen seinen Bedürfnissen, daher sollten die Angehörigen als nachgeordnet betroffene Patienten betrachtet und auch entsprechend in die Betreuung einbezogen werden. In der randomisierten kontrollierten klinischen Studie einer phasenspezifischen evidenzbasierten Psychoedukation und TC-Intervention wurden 244 Patienten-Partner-Paare eingeschlossen. Es wurden drei Interventionsgruppen gebildet: Standardisierte Psychoedukation (SE), TC und SE+TC. Die Kontrollgruppe erhielt das konventionelle Prozedere der Betreuung (Disease Management=DM). Die phasenspezifische standardisierte Psychoedukation und Telephonkonsultation wurden aufbauend auf den Modellen „Stress und Verarbeitung“ und „Krise“ entwickelt. Ziel war, die körperlichen, emotionalen und sozialen
Anpassungen in jeder der vier Phasen zu maximieren indem eine positive Einschätzung der Diagnose- und Therapiebezogenenen Ereignisse gefördert wurde. Die speziellen Bedürfnisse sowohl von Patienten als auch deren Partnern, die durch TC angesprochen werden, sind die Wahrnehmung der Einschätzung von Schlüsselereignissen und Besorgnissen, die sich auf eine bestimmte Phase beziehen, zu erfassen, Fragen über medizinische Behandlungen, Prozeduren und Nebeneffekte zu klären, die Angemessenheit der sozialen Unterstützung zu erforschen und die Wirksamkeit von Bewältigungsstrategien und sozialer Unterstützung zu erleichtern.

\section{Ergebnisse}

Unabhängig in welcher Interventionsgruppe die Studienteilnehmer waren, wurden signifikante Hauptwirkungen über die Zeit sowohl für Patienten wie für Partner festgestellt. Die Patientenwerte verbesserten sich über die Dauer der Untersuchung in psychologischem Wohlbefinden, Schwere der Nebenwirkungen, der wahrgenommenen Gesamtgesundheit und der sozialen Anpassung. Die Partnerwerte verbesserten sich über die Zeit bei den körperlichen Symptomen und der sozialen Anpassung im Arbeitsund gesellschaftlichen Umfeld. Allerdings zeigte sich, dass der Einsatz von Videovermittelter Psychoedukation eine Verbesserung des psychischen Wohlbefindens von der postoperativen Phase bis zur Genesung erzielte, während TC alleine zwar postoperativ eine signifikante Besserung brachte, in der Phase von adjuvanter Therapie zur Genesung jedoch einen Abfall der Werte aufzeigte. Möglicherweise, so vermuten die Autoren, bestand aber auch bei der TC viel stärker die Möglichkeit persönliche Sorgen und Ängste zu äußern, sodass Anpassungsprobleme offener berichtet wurden. Frauen, die individualisierte TC erhielten, berichteten positiv über die Möglichkeit tiefliegende Ängste und Anliegen teilen zu können, die sie in einer Gruppensituation nicht geteilt hätten. Die einzige Gruppe, die hinsichtlich der Belastung durch und Schwere der Ne- benwirkungen eine Reduktion zeigte, war jene mit kombinierter Intervention SE+TC, was möglicherweise darauf zurückzuführen ist, dass die Patienten ihre Nebenwirkungen besser einschätzen können und sie damit weniger schwer und belastend empfinden und dass sie auch bessere Bewältigungsstrategien einsetzen als jene Patienten, die nur eine Intervention oder die Standardbehandlung erhielten. Die Wahrnehmung der Gesamtgesundheit zeigte in allen Interventionsgruppen in allen Phasen signifikante Verbesserungen und auch die Werte der Partner für diesen Aspekt waren ziemlich konstant über den Zeitverlauf. Die Situation in der sozialen Umgebung verbesserte sich für Patienten graduell während des Zeitverlaufs, während sie für die Partner vor allem in der Zeit nach der adjuvanten Therapie und während der Genesungsphase besser wurde.

Die Rubrik pflege\&wissenschaft erfolgt in Kooperation mit dem Institut für Pflegewissenschaft der Medizinischen Universität Graz.

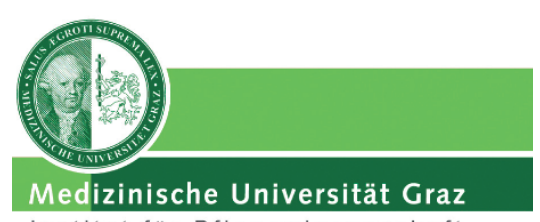

Institut für Pflegewissenschaft

Fazit

Obwohl die Interventionen Besserungen gebracht haben, zeigt sich doch, dass keine Intervention für alle Aspekte besser war. Daher wird es, so die Autoren, notwendig sein, gezieltere Interventionen für Frauen mit Brustkrebs zu entwickeln, um ihre Bedürfnisse in jeder der vier Phasen der Brustkrebserkrankung bestmöglich erfüllen zu können.

Wendy C. Budin, Carol Noll Hoskins, Judith Haber, Deborah Witt Sherman, Greg Maislin, Jacqueline R. Cater, Frances Cartwright-Acarese, quildred Ortu Kowalski, Christina Beyer McSherry, Renee Fuerbach, Shilpa Shukla: Breast Cancer. Funcitoningh, Counseling, and Adjustment Among Patients and Partners: A Randomized Clinical Trial, in: Nursing research, Max/June 2008, Vol. 57, No 3: 199- 213 